\title{
Comunicación
}

\section{Schistosomus reflexus en un feto bovino en Colombia: reporte de caso}

\author{
Schistosomus reflexus in a bovine fetus in Colombia: a case report \\ Cesar Augusto Gómez Velásquez ${ }^{1}$, Gabriel Andrés Pinilla Peña ${ }^{2,3}$, \\ Juan David Córdoba Parra ${ }^{1}$
}

\section{Resumen}

Se reporta un caso de Schistosomus reflexus ocurrido en una ganadería en Maní, Casanare, Colombia. El motivo de consulta fue una vaca con distocia, a la cual se le realizó examen obstétrico por palpación transvaginal, reportándose una malformación del ternero con exposición de vísceras abdominales. Se procede a realizar una cesárea y se extrae una cría muerta con inversión espinal, cierre incompleto de la pared ventral abdominal, exposición de vísceras abdominales y artrogriposis, compatible con Schistosomus reflexus. Este es el primer caso reportado de esta deformidad en bovinos en Colombia.

Palabras clave: anomalía; cesárea; congénito; feto; obstetricia

\section{Abstract}

A case of Schistosomus reflexus occurred in a cattle farm in Maní, Casanare, Colombia is reported. The reason for consultation was a cow with dystocia, who underwent obstetric examination by transvaginal palpation, reporting a malformation of the calf with exposure of abdominal viscera. A cesarean was performed and a dead calf with spinal inversion was extracted, the calf had incomplete closure of the abdominal ventral wall, exhibition of abdominal viscera and arthrogryposis, compatible with Schistosomus reflexus. This is the first reported case of this deformity in cattle in Colombia.

Key words: anomaly; caesarean section; congenital; fetus; obstetrics

${ }^{1}$ Programa de Medicina Veterinaria, Facultad de Ciencias Agropecuarias, Universidad de la Salle, Bogotá, Colombia

${ }^{2}$ Universidad de la Salle, Bogotá, Colombia

${ }^{3}$ E-mail: gpinilla47@unisalle.edu.co

Recibido: 30 de enero de 2018

Aceptado para publicación: 18 de julio de 2018 


\section{INTRODUCCIÓN}

Schistosomus reflexus es una malformación congénita poco frecuente y que principalmente se presenta en rumiantes (Laugthon et al., 2005; Prestes y Megid, 2010; Ozalp et al., 2011). También se ha reportado en otras especies, incluyendo equinos (Irwin y Pulley, 1975), porcinos (Reyes et al., 2006), felinos (Bertolo et al., 2017), caninos (Molina et al., 2012; Bertolo et al., 2017), tortugas (Barcenas-Ibarra et al., 2016), rinocerontes (Lankton et al., 2014) y camellos (Elias, 1991). Se caracteriza principalmente por presentar un cierre incompleto de la pared ventral abdominal y/o torácica ocasionando la exposición de las vísceras abdominales y por presentar una inversión de la columna vertebral quedando el cráneo paralelo al sacro y los miembros posteriores. En algunos casos se puede observar que los miembros presentan artrogriposis (Laugthon et al., 2005).

Schistosomus reflexus es considerado como una forma severa de hernia abdominal asociada con problemas esqueléticos que surgen en la vida embrionaria temprana cuando los bordes laterales del disco germinal se reflejan dorsalmente, en lugar de ventralmente, para formar las cavidades corporales (Dennis y Mayer 1965). En bovinos, se sospecha de un modo de herencia autosómico recesivo debido a los grupos de casos observados en la descendencia de ciertos toros (Citek, 2012; Ferreira et al., 2013). La causa de Schistosomus reflexus sigue siendo ambigua; sin embargo, se estima que los factores genéticos y la interacción de múltiples genes podrían influir en la aparición de tal defecto (Jana y Ghosh, 2001). También se asocia a factores genéticos, agentes infecciosos y factores ambientales o una combinación de todos estos (Kumar et al., 2017).

En una revisión retrospectiva de 6901 distocias en bovinos, atendidas por 21 veteri- narios durante un periodo de 20 años (19661985), 90 casos (1.3\%) fueron causados por Schistosomus reflexus, donde la mayoría de estos casos $(56.7 \%)$ fueron tratados por fetotomía (Knight, 1996). Otros autores también reportan que la distocia causada por Schistosomus reflexus fue resuelta por medio de fetotomía o por cesárea (Padma et al., 1993; Prestes y Megid, 2010; Jana D y Jana M, 2013).

\section{Reporte de Caso}

El 22 de febrero de 2013, en una ganadería doble propósito ubicada en Mani, Casanare, Colombia, se presenta una vaca mestiza de cinco años con un problema de distocia. La vaca estaba en su segunda gestación de aproximadamente 250 días y en proceso de aborto. La vaca presentaba contracciones propias del parto, aunque improductivas, y exposición de membranas fetales por la comisura vulvar.

Los signos vitales de la vaca estaban dentro de los valores de referencia, presentando una frecuencia cardiaca de $83 \mathrm{lpm}$, frecuencia respiratoria de $28 \mathrm{rpm}$, temperatura corporal de $38.5^{\circ} \mathrm{C}$ y condición corporal de 3.5/5.0. Al realizar el examen obstétrico se palpan estructuras viscerales del feto y se identifica un feto deforme con las lesiones características de la malformación en cuestión, por lo cual se hace un diagnóstico presuntivo de Schistosomus reflexus.

Se decide realizar la tracción moderada del feto para hacer una extracción por vía vaginal, pero sin éxito, por lo que se decide realizar una cesárea por laparotomía abdominal por la fosa paralumbar izquierda, logrando sacar el feto deforme. Se confirma el diagnóstico de Schistosomus reflexus con la descripción de las alteraciones del feto (Figuras 1 y 2). 


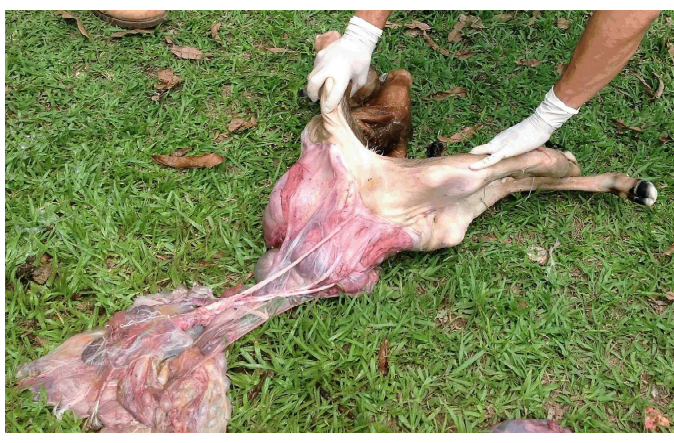

Figura 1. Exposición de las vísceras abdominales por cierre incompleto de la pared abdominal ventral, presentación de artrogriposis de los cuatro miembros y anquilosis de las articulaciones distales de los miembros

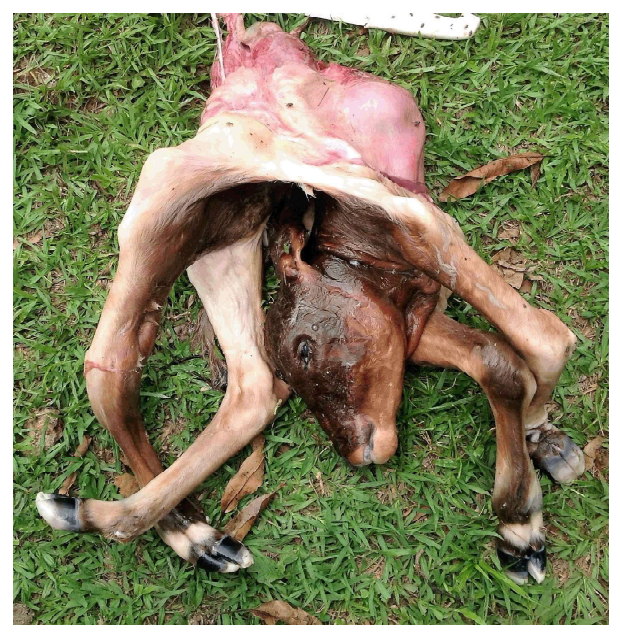

Figura 2. Inversión completa de la columna vertebral quedando el cráneo paralelo al sacro, presentación de artrogriposis de los cuatro miembros, cierre incompleto de la pared abdominal.

\section{Discusión}

Schistosomus reflexus ha sido reportado en caninos en Colombia (Molina et al., 2012), pero este es el primer reporte en bovinos. No obstante, existen diversos reportes en rumiantes en otros países (Prestes y Megid, 2010; Ferreira et al., 2013; Barua et al., 2014; Sitali et al., 2014).
La resolución del problema por cesárea ha sido ampliamente reportada para poder extraer el feto deforme (Padma et al., 1993; Jana y Ghosh, 2001; Jana D y Jana M, 2013). El feto extraído presentó exposición de las vísceras abdominales por cierre incompleto de la pared abdominal ventral, presentación de artrogriposis de los cuatro miembros y anquilosis de las articulaciones distales de los miembros e inversión completa de la columna vertebral, quedando el cráneo paralelo al sacro, lo cual ocurre en todos los casos de Schistosomus reflexus (Laugthon et al., 2005). Así mismo, se reporta pulmones hipoplásicos (Ozcan et al., 2003; Laugthon et al., 2005), ausencia de unión de las sínfisis púbicas (Laugthon et al., 2005), esternón hendido (Bedford, 1967) y muchas otras deformidades.

\section{Literatura Citada}

\section{Bárcenas-Ibarra A, Rojas-Lleonart} I, Lozano-Guzmán RI, García-Gasca A. 2016. Schistosomus reflexus syndrome in olive ridley sea turtles (Lepidochelys olivacea). Vet Pathol 54: 171-177. doi: 10.1177/0300985816651682

2. Barua M, Hossain F, Islam MZ, Islam K. 2014. Schistosomus reflexus syndrome, a congenital defect in ruminants; occurrence and management through caesarean section in dairy cow. Res J Vet Pract 2: 40-41. doi: 10.14737/ journal.rjvp/2014/2.3.40.41

3. Bedford P. 1967. Schistosomus reflexus in a goat: a case report. Vet Rec $80: 326$

4. Bertolo L, Conceição, M. E., Aguirre L, Martins D. Macedo B, Coutinho L, Cardoso A, Pereira W. 2017. Schistosomus reflexus in a dog and a cat. Acta Sci Vet 45: 1-4.

5. Citek J. 2012. Pedigree analysis of Czech Holstein calves with Schistosoma reflexum. Acta Vet Scand 52: 22. doi: 10.1186/1751-0147-54-22 
6. Dennis SM, Mayer EP. 1965. Schistosomus reflexus in a sheep. Vet Rec 47: 1386-1387.

7. Elias E. 1991. Left ventrolateral cesarean section in three dromedary camels (Camelus dromedarius). Vet Surg 20: 323-325. doi: 10.1111/j.1532950X.1991.tb01277.x

8. Ferreira DO, Santarosa BP, Monteiro-Toma CD, Belotta AF, Chiacchio SB, Gonçalves RC, et al. 2013. Estudo anatomorfológico, radiográfico e tomográfico de Schistosomus reflexus em ovino da raça Dorper: relato de caso. Arq Bras Med Vet Zoo 65: 1096-1102.

9. Irwin M, Pulley L. 1975. Schistosomus reflexus in an equine fetus. Vet Med Sm Anim Clin 70: 44-45.

10. Jana D, Ghosh M. 2001. Dystocia due to foetal monster with Schistosomus reflexus and ectopic viscera - a case report. Indian Vet J 78: 333 - 334.

11. Jana D, Jana M. 2013. Studies on Schistosomus reflexus in indigenous cattle in tropical west bengal, India. Exploratory Anim Med Res 3: 74-77.

12. Knight RP. 1996. The occurrence of Schistosomus reflexus in bovine dystocia. Aust Vet J 73: 105-107. doi: 10.1111/j.1751-0813.1996.tb09988.x

13. Kumar A, Kumar S, Gunwant P, Verma A, Phogat JB. 2017. Schistosomus reflexus monster fetus in bovine and its successful management. Res J Vet Pract 5: 25-27.

14. Lankton JS, VanderHart DJ, Terrell SP. 2014. Schistosomus reflexus-like malformation in a southern white rhinoceros (Ceratotherium simum simum). J Zoo Wildlife Med 45: 708-711. doi: 10.1638/2013-0272R1.1
15. Laugthon KW, Fisher KR, Halina WG, Partlow GD. 2005. Schistosomus reûexus syndrome: a heritable defect in ruminants. Anat Histol Embryol 34: 312318. doi: $10.1111 /$ j. 1439-0264.2005.00624.x

16. Molina VM, Oviedo CA, Casado A, Arias MP. 2012. Schistosomus reflexus en un canino: reporte caso. Rev Med Vet Zoot 59: 49-55.

17. Ozalp GR, Celikler S, Simsek G, Ozyigit MO, Inan S. 2011. A case of Schistosoma reflexum in a cat with chromosomal aberrations. Reprod Domest Anim 46: 373-376. doi: 10.1111/ j.1439-0531.2010.01649.x

18. Ozcan K, Tuzcu M, Erginosoy $S$, Ozturkler Y. 2003. Schistosomus reflexus in cattle in kars province. Indian Vet J 80: 693 - 694.

19. Padma Rao VP, Ramachandraiah $K$, Reddy AR, Subbaiah DV, Chandrasekhar B. 1993. Schistosomus reflexus in a monocephalus tripus dibrachius calf. Indian Vet J 70: 1083.

20. Prestes NC, Megid J. 2010. Uma forma rara de ocorrencia de Schistosomus reflexus em bovino: relato de caso. Vet Zootec 17: 214-218.

21. Reyes IR, Pérez F, Reyes LE. 2006. Schistosoma reflexus incompleto en una cerdita mestiza Criollo de Cuba X Yorkshire. Caso Clínico. REDVET 7(5). [Internet]. Disponible en: http:// www.veterinaria.org/revistas/redvet/ n050506/050604.pdf

22. Sitali MC, Mwaanga ES, Zulu VC, Mwanza AM. 2014. Schistosomus reflexus from a Holstein-Friesian cowcase report. Theriogenology Insight 4: 65-70. doi: 10.5958/2277-3371.2014.00735.9 